\title{
Hemoglobinúria paroxística noturna (HPN) em paciente pediátrico: relato de caso
}

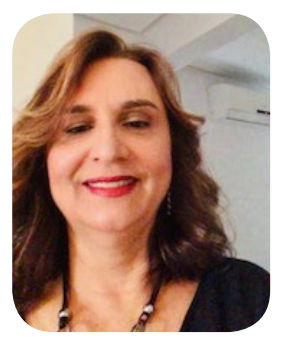

\section{INTRODUÇÃO}

A Hemoglobina Paroxística Noturna (HPN) é uma doença hematológica rara, clonal e adquirida, caracterizada por anemia hemolítica intravascular, episódios trombóticos e pancitopenia (graus variáveis de insuficiência medular) ${ }^{1}$.

Desordem clonal, causada por mutação somática adquirida do gene PIG-A (fosfatidilinositol glicano da Classe A), ligado ao cromossomo $X$, afeta a célulatronco hematopoiética responsável pela produção de todas as células do sangue ${ }^{1}$.

Essa mutação resulta na deficiência de várias proteínas ancoradas pela GPI (glicofosilfosfatidilinositol), entre elas o D55 e o D59, inibidores do sistema de complemento, levando à destruição dos eritrócitos deficientes em GPI, bem como ativação dos leucócitos e plaquetas, distúrbios da coagulação e fibrinólise. Existe relação entre HPN e a anemia aplástica (AA) ${ }^{31}$.

Devido à raridade da doença, os dados epidemiológicos são escassos, com incidência estimada de 0.3100.000 habitantes-ano e a prevalência de 1,59 casos por 100.000 habitantes e na faixa etária pediátrica, na qual corresponde a $10 \%$ da já rara incidência em adultos (de 1 em 10^5 a 1 em 10^6) ${ }^{2,32}$.

\section{MÉTODOS}

Relato de caso no qual as informações foram obtidas por meio de revisão do prontuário, registro dos métodos diagnósticos utilizados e revisão da literatura.

\section{RELATO DE CASO}

Paciente do sexo feminino, 14 anos, deu entrada no ambulatório apresentando colúria e anemia, sem hepatoesplenomegalia, febre, dores osteoarticulares ou linfadenomegalia. Há 3 meses da admissão relata quadro semelhante (colúria+ palidez) tratado por suspeita de infecção urinária, não confirmada pela cultura, e liberada para investigação ambulatorial, apesar de queda dos níveis de $\mathrm{HB}$ e níveis elevados de $\mathrm{LDH}^{7}$

Antecedente de Transtorno Depressivo Maior há 1 ano, com acompanhamento psiquiátrico em uso de sertralina.

Diante do quadro foi afastada anemia hemolítica hereditária ou adquirida (imunológica), porém com persistência da anemia acompanhada de adinamia, fraqueza, cansaço, tonturas e mal-estar, e sendo excluída a possibilidade de doença reumatológica/inflamatória. ${ }^{4}$

Foi realizado mielograma e biópsia de medula óssea, cuja imunofenotipagem do sangue periférico e medular detectou alteração clonal que confirmou diagnóstico de Hemoglobinúria Paroxística Noturna (HPN).

Paciente foi encaminhada para Serviço de Referência no tratamento da doença.

\section{RESULTADOS}

\section{Imunofenotipagem de MO:}

Observada ausência de expressão dos marcadores CD16 em granulócitos, CD14 e CD300 em monócitos. A expressão destes marcadores depende do estágio maturativo celular e da presença de GPI (glicofosfaditilinositol).

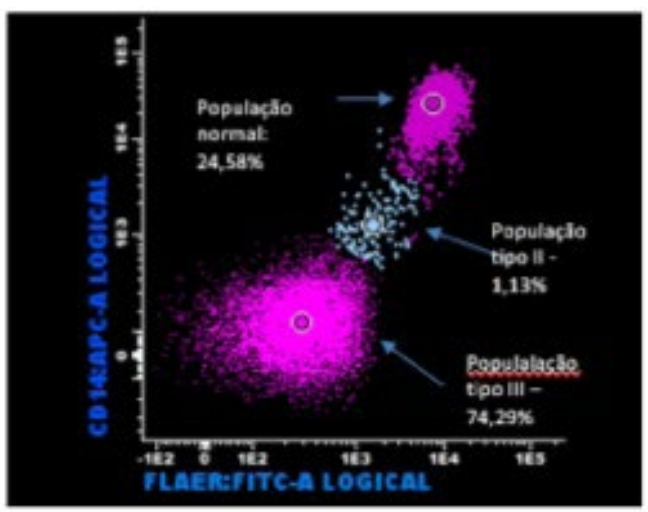



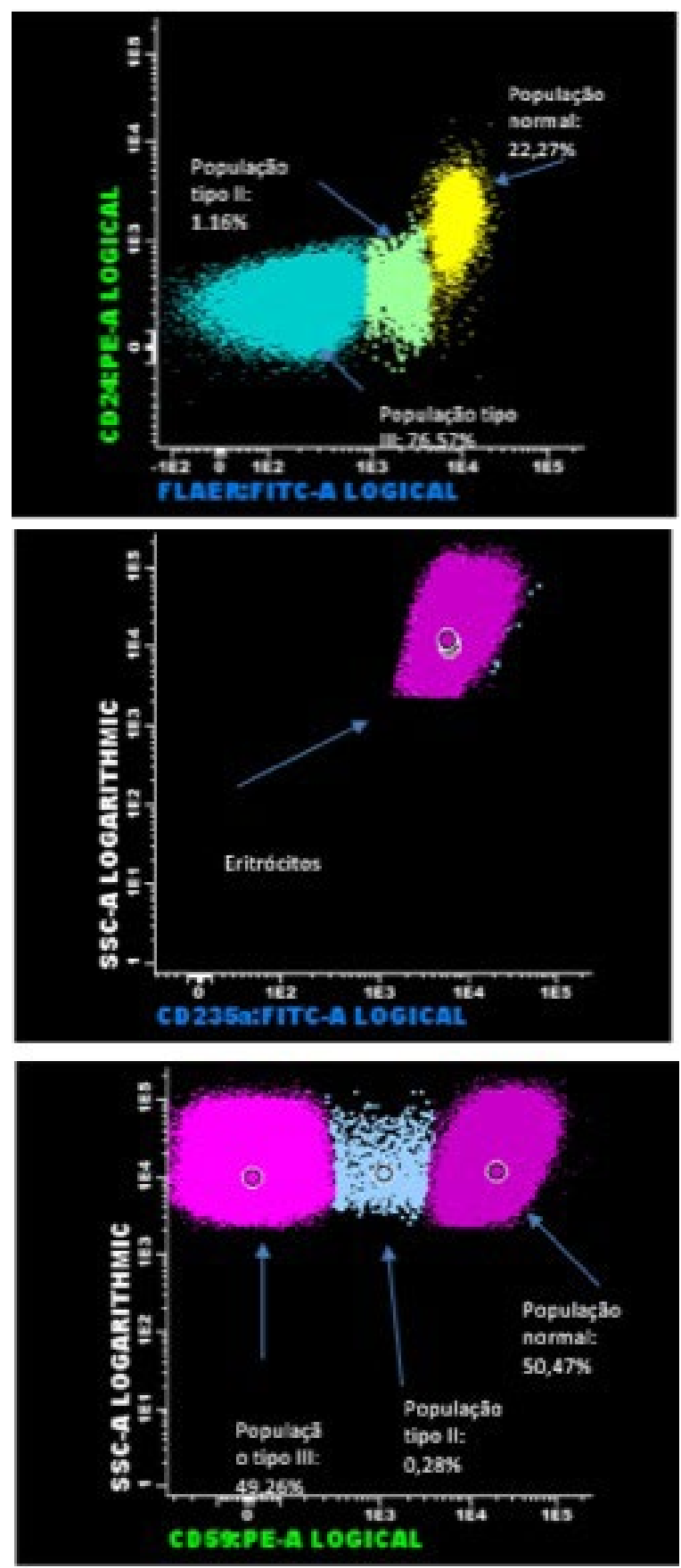

Figura 1 - Resultado de marcadores celulares, pesquisa de clone HPN: 13.

A análise por citometria de fluxo mostrou perda de expressão de antígenos ligados a GPI em granulócitos, monócitos e hemácias 16. Presença de clone HPN na amostra analisada.

\section{DISCUSSÃO}

A HPN é uma anemia hemolítica crônica, adquirida, rara, de curso clínico extremamente variável. Possui idade média de diagnóstico de 40 anos e tempo médio de sobrevivência de 10 anos, afetando homens e muIheres na mesma proporção. ${ }^{3}$

Apresenta-se frequentemente em associação com outras doenças hematológicas, especialmente com síndromes de falência medular, como anemia aplásica e síndrome mielodisplásica. ${ }^{14}$ É considerada ainda um tipo de trombofilia adquirida, apresentando-se com tromboses venosas variadas, sua maior causa de mortalidade. ${ }^{4,13}$

A etiologia da trombose é multifatorial, potencialmente fatal , podendo ocorrer mesmo com uso de anticoagulantes. ${ }^{3}$

A mutação ocorre no gene da fosfaditilinositolglica na classe-A e resulta no bloqueio precoce da síntese de âncoras de glicosilfosfaditilinositol (GPI), responsáveis por manter aderidas à membrana plasmática dezenas de proteínas com funções específicas, controlando a ativação da cascata de complemento. ${ }^{3}$

A sintomatologia da doença pode ser variada: como adinamia, dor abdominal, espasmo esofágico, dor a deglutição e dor torácica, disfunção erétil e possível trombose ${ }^{4}$ e como complicações mais tardias, doença renal crônica e hipertensão pulmonar., ${ }^{1,4-8}$

A tríade anemia hemolítica (hemoglobinúria) + pancitopenia com infecções + tromboses 12 faz da HPN uma síndrome clínica única, que passou a ser encarada como um defeito mutacional clonal da célula-tronco hematopoética (CTH). Segue abaixo, na Tabela 1, alterações que sugerem a investigação para HPN.

Tabela 1 - Alterações clínicas e laboratoriais.

\begin{tabular}{l} 
PACIENTES DE ALTO RISCO PARA A \\
INVESTIGAÇAO DE HPN \\
Anemia hemolítica com ou sem hemoglobinúria, com hemácias \\
de aparência normal e sem outras etiologias \\
\hline Hemólise adquirida com teste de Coombs negativo \\
\hline Hemólise adquirida sem esplenomegalia \\
\hline \hline Neutropenia, plaquetopenia, hipoplasia ou aplasia de medula \\
óssea, associadas à hemólise \\
\hline \hline Trombose venosa recorrente, principalmente em sítios pouco \\
usuais (hepática ou mesentérica) \\
\hline \hline Dor abdominal recorrente ou sintomas neurológicos associados \\
a citopenias \\
\hline \hline
\end{tabular}

Adaptado Hemorio, 2018, 14. 
Os sintomas apresentados com maior frequência foram os relacionados à anemia. Todos os pacientes, além de anemia severa, também evidenciavam leucopenia e plaquetopenia. A maioria apresentava LDH elevado. As maiores causas de morte dos pacientes com HPN são trombose, infecção e doenças malignas, podendo ainda ocorrer hemorragia fatal nos pacientes plaquetopênicos. ${ }^{18,19}$ Para uma análise adequada do clone HPN, recomenda-se a avaliação com citometria de fluxo em três linhagens: eritrócitos, granulócitos e monócitos.

Com marcadores preconizados para análise de CD59 em eritrócitos, CD14 e FLAER para monócitos, como CD24 e FLAER em granulócitos, como realizado no relato de caso apresentado. ${ }^{16}$

Essas metodologias de testes diagnósticos para HPN foram validadas na prática clínica, inclusive no Brasil. ${ }^{15}$

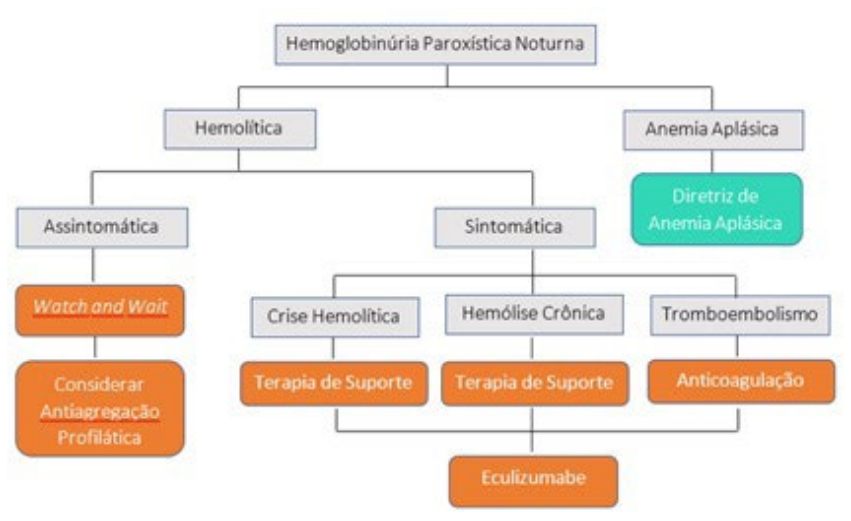

Figura 2 - Algoritmo do Tratamento de HPN (29).

\section{TRATAMENTO}

As principais modalidades de tratamento para HPN são: terapia de suporte, corticosteroides e andrógenos; inibição do sistema de Complemento e transplante alogênico de células-tronco hematopoiéticas. $O$ tratamento é instituído de acordo com as manifestações clínicas da doença.

Transfusão de hemocomponentes e reposição de ácido fólico e ferro são frequentemente necessárias. ${ }^{17}$ Corticoide em baixas doses diminui a taxa de hemólise em alguns pacientes.

Androgênios podem ser usados em pacientes com anemia, sendo descrita ainda moderada elevação de plaquetas. ${ }^{20}$

O uso de Eritropoetina é considerado nos casos de anemia com reticulopenia $(<100.000)$. Há relatos de uso de imunossupressores. O uso de anticoncepcional oral deve ser evitado em virtude de estar associado a aumento do risco de trombose.

Trombólise precoce, seguida de anticoagulação por longo tempo, reverte ou diminui as tromboses para estágios de menor comprometimento. ${ }^{18}$

Atualmente, a terapia é baseada na inibição do sistema complemento através da utilização do Eculizumabe, um anticorpo monoclonal humanizado que bloqueia a decomposição de C 5 em C5a e C5b, através da ligação à proteína do sistema de complemento C5. ${ }^{19,21-24}$

Os pacientes em tratamento com o anticorpo monoclonal Eculizumabe apresentaram melhora dos sintomas, normalização da hemoglobina e diminuição dos níveis de DHL, deixando-os livres de anemia e transfusões, tão bem como redução dos riscos de danos a órgãos decorrentes da hemólise e da incidência de trombose, insuficiência renal e hipertensão pulmonar, melhorando significativamente a qualidade de vida e reduzindo o risco de mortalidade com o aumento da sobrevida. ${ }^{10,11,17,19,21-25}$

Existem diretrizes internacionais disponíveis a respeito de educação e estudos de HPN, que indicam o uso de Eculizumabe, em conjunto com terapia de suporte, já citada anteriormente..$^{17,28-29}$

Tabela 2 - Recomendações do uso de Eculizumabe

RECOMENDAÇÕES DE USO DE ECULIZUMABE

\begin{tabular}{l} 
Pacientes com anemia hemolítica intravascular crônica que \\
apresentem atividade de $\mathrm{DHL} 1,5$ vez acima do limite superior \\
\hline Pacientes com trombose atribuível a HPN \\
\hline \hline Pacientes com insuficiência renal atribuída à HPN \\
\hline \hline Pacientes com necessidade de transfusões regulares \\
\hline \hline Pacientes com insuficiência pulmonar \\
\hline Pacientes com dor intensa relacionada à musculatura lisa \\
\hline \hline
\end{tabular}

Villegas A, Gaya A, et. al. Med Clin 2016, 28.

Estudos realizados de longa duração demonstram a segurança e tolerabilidade no uso do Eculizumabe. ${ }^{23}$ No entanto, o transplante alogênico de medula óssea é o único tratamento curativo disponível, embora esteja por si mesmo associado a altas taxas de morbimortalidade.

O risco de mortalidade de $32-42 \%$ e de desenvolvimento de doença do enxerto contra o hospedeiro (DECH) é cerca de $50 \% .{ }^{26,27}$

Em diretrizes internacionais, Alemanha e Espanha indicam o uso do Eculizumabe, em conjunto com terapias de suporte. ${ }^{28,29}$ 


\section{CONCLUSÃO}

A HPN apresenta um curso clínico desfavorável, devido as suas complicações e dificuldade de se instituir um tratamento específico. Estima-se que na história natural da HPN, cerca de $50 \%$ dos pacientes morrem em 10 anos..$^{1,31}$

A introdução do tratamento específico com inibidor de complemento permite que os níveis de mortalidade se assemelhem à população sem a doença, porém devido a custos, ainda no Brasil, não é factível a todos os pacientes aos quais são indicados. ${ }^{10,11} \mathrm{Na}$ infância, devido a sua raridade, ainda não existem protocolos específicos para o início da terapia. ${ }^{32}$

O diagnóstico precoce ainda se traduz na melhor estratégia para redução de morbimortalidade através da terapia de suporte, evitando complicações tardias, permitindo, também, um melhor entendimento da doença, servindo de estímulo para novas pesquisas, a fim de encontrarmos novas terapias, que possam ser instituídas e liberadas para todos os pacientes, sejam adultos ou crianças e que sejam viáveis economicamente, já que o transplante alogênico apresenta-se com altos índices de mortalidade, estando indicado somente em situações específicas. ${ }^{26,27}$

\section{REFERÊNCIAS}

1- Hilmen P, Lewis et. al. Natural history of paroxysmal nocturnal hemoglobinúria .N EnglJ Med.1995.

2- Hill A,Platts PJ et. al. The Incidence and Prevalence of paroxysmal nocturnal hemoglobinúria $(\mathrm{PNH})$ and Survival of Patients in Yorkshire .Blood.2006,108:985.

3- Hill A, Kelly R JHilmen P.Trombosis in paroxysmal nocturnal hemoglobinúria. Blood.2013, June.

4- Hill A, et. al.: Paroxysmal nocturnal hemoglobinúria, Nat Rev Dis Prim 2017.

5- Socie G, et. al. Paroxysmal nocturnal hemoglobinúria long term follow upand prognostic factors.French society of Haematology, Lancet, London 1996.

6- Lee JW, Jang JH et. al.: Clinical signs and symptoms associated with increased risk for thrombosis in patients with paroxysmal nocturnal hemoglobinúria from a Korean Registry.Int J Hematol 2013.

7- Jang JH, Kim Js, et. al. .Predictive Factors of Mortality in population of patients with Paroxysmal nocturnal hemoglobinúria $(\mathrm{PNH})$. Results from a korean $\mathrm{PNH}$ Registry.J Korean Med 2016.

8- Weitz I,Meyers G, et. al., Cross-sectional validation study of patient-reported outcomes in patients with Paroxysmal nocturnal hemoglobinúria. Intern Med $\mathrm{J}$. 2013,43 (3):298-307.

9- De Latour R P, Mary JY et. al. Paroxysmal noctur- nal hemoglobinúria. Natural history of disease and subcategories. BLOOD 2008.

10- Loschi M, Porcher R, et al. Impact of Eculizumab treatment of Paroxysmal nocturnal hemoglobinúria: a treatament versus no-treatment study. Am J Hematol 2016.

11- Kelly $\mathrm{R} \mathrm{J}$, Hill A, et. al. Long term treatment with eculizumab of Paroxysmal nocturnal hemoglobinúria: sustained efficacy and improved survival CME article, Blood 2011,117 (25) 6786-92.

12- De Azambuja AP, Malvezzi M, Pasquini R et. al. Paroxysmal nocturnal hemoglobinúria clone in 103 Brazilian Patients: diagnosis and classification. Rev Bras Hematol Hemoter 2015.

13- Borowitz MJ , Craig FE et. al. .Guidelines for the diagnosis and monitoring of Paroxysmal nocturnal hemoglobinúria and related disorders by flow cytometry. Cytom part b - Clin Cytom 2010.

14- Hemorio. Hemoglobinuria Paroxistica Noturna, Protocolos 2018.

15- Morado M, Freire Sandes A, et. al. Diagnostic screening of Paroxysmal nocturnal hemoglobinúria: Prospective multicentric evaluation of the current medical indications. Cytom part b - Clin Cytom 2017.

16- Sutherland DR, Keeney M. Pratical Guidelines for the High sensitivity detection and monitoring of $\mathrm{Pa}-$ roxysmal nocturnal hemoglobinúria: clones by flow cytometry. Cytom part b - Clin Cytom 2012.

17- Sahin F, Akay O M et. al., Pesg PNH diagnosis, follow up and treatment guidelines. Am J Blood 2016-6 (2) $19-27$.

18- Hall C, Richards S, Hilmen P. Primary Profilaxy with warfarin prevents thrombosis in Paroxysmal nocturnal hemoglobinúria (PNH )BLOOD 2003.

19- Hillmen $P$, Muus $P$,et at. Effect of the complement inhibitor eculzumab on thromboembolism in patients with Paroxysmal nocturnal hemoglobinúria.Blood 2001

20- Issaragrisil S, Piankjagun A et at .Corticosteroids therapy in Paroxysmal nocturnal hemoglobinúria.Am j hematol 1987, 25 (1):77-83.

21- Hillmen $P$,Hall $C$,et. al. Effect of Eculizumab on Hemolysis and Transfusion Requiements in Patients with Paroxysmal nocturnal hemoglobinúria. $\mathrm{N}$ Engl $\mathrm{J}$ Med 2006355 (12) 1233-43.

22- Hillmen P, Young NS, et. al. The complement Inhibitor Eculizumab Paroxysmal nocturnal hemoglobinúria. N Engl J Med 2006,355(12 ) 1233-1243.

23- Hillmen $P$, Muus $P$, et. al. Long term safety and efficacy of sustained eculizumab for the treatment of $\mathrm{Pa}$ roxysmal nocturnal hemoglobinúria, $\mathrm{Br} \mathrm{J}$ Haematology 2013. 
24- Brodsky RA et. al. Multicenter phase 3 study of the complement Inibitor eculizumab for the treatment of patients with Paroxysmal nocturnal hemoglobinúria. Blood 2008-111 (4)1840-7.

25- Socié $\mathrm{G}$ et. al. Changing Prognosis in Paroxysmal nocturnal hemoglobinúria Diseases subcategories: an analysis of de International PNH Registry. Intern Med J 2016.

26- Santarone S, Bacigalupo et. al. Hematopietic stem cell transplantation for Paroxysmal nocturnal hemoglobinúria. Long term results of a retrospective study on behalf of de gruppo italiano trapianto midollo osseo (GITMO).

27- De Latour RP,et. al. Allogeneic stem cell transplantation in Paroxysmal nocturnal hemoglobinúria.Haematologica 2012.

28- Villegas A, Gaya A et. al. Spanish consensus statement for diagnosis and treatment of Paroxysmal nocturnal hemoglobinúria. Med Clin 2016.

29- Schubert J, et. al. Paroxysmal nocturnal hemoglobinúria $(\mathrm{PNH})$ GUIDELINE: recommendations from the society for diagnosis and therapy of haematological and oncological diseases. Onkopedia 2012.

30- Almeida AM, Bedrosian C et. al. Clinical Benefit of Eculizumab in patients with transfusion history in the International Paroxysmal nocturnal hemoglobinúria. Hemoglobinuria Registry. Intern Med J 2017.

31- Martha Mariana de Almeida Santos Arruda, Celso Arrais Rodrigues, Mihoko Yamamoto, Maria Stella Figueiredo. Trabalho realizado na Disciplina de Hematologia e Hemoterapia da Universidade Federal de São Paulo - UNIFESP, São Paulo, SP hemoglobinúria paroxística noturna: da fisiopatologia ao tratamento.

32- Russel E, Ware M D, et al. Paroxysmal nocturnal hemoglobinúria with onset in childhood and adolescence. The New England Journal of Medicine, 325 (14) 991.

1- Serviço de Hematologia Pediátrica do HSI

2-Acadêmicos do Curso de Medicina

3- Serviço de Onco-hematologia do HSI

Endereço para correspondência:

jansamp@terra.com.br 